\title{
Diel changes in the egg production rate of Acartia tonsa (Copepoda, Calanoida) and related environmental factors in two estuaries
}

\author{
D. E. Stearns ${ }^{1,2, *}$, P. A. Tester ${ }^{3, * *}$, R. L. Walker \\ ${ }^{1}$ Skidaway Institute of Oceanography, Savannah, Georgia 31416-0687, USA \\ ${ }^{2}$ Marine Environmental Sciences Consortium, Dauphin Island Sea Lab, Dauphin Island, Alabama 36528, USA \\ ${ }^{3}$ National Marine Fisheries Service, NOAA, Southeast Fisheries Center, Beaufort Laboratory, Pivers Island, Beaufort, \\ North Carolina 28516-9722, USA \\ ${ }^{4}$ Marine Extension Service, University of Georgia, Savannah, Georgia 31416-0687, USA
}

\begin{abstract}
Two $48 \mathrm{~h}$ field experiments were completed in different USA east coast estuaries to study short-interval changes in egg production by the coastal copepod Acartia tonsa Dana. In April and June 1986, adult females were collected periodically, sorted into separate glass vials filled with $20 \mu \mathrm{m}$ or $202 \mu \mathrm{m}$ filtered seawater, then placed near the estuarine bottom during the day and at $1 \mathrm{~m}$ depth at night to mimic the vertical spatiotemporal location of the adult A.tonsa population. Field measurements of the following factors were made at $3 \mathrm{~h}$ intervals: (1) egg production; (2) light intensity; (3) water temperature; (4) salinity; (5) concentrations of plant pigments and (6) A tonsa gut fullness measured fluorometrically from field-collected, nonexperimental specimens. Copepods in both experiments showed strong nocturnal egg-laying behavior Changes in temperature, salinity or plant pigments did not explain this behavior, but egg production correlated well with gut fullness measured 24 to $27 \mathrm{~h}$ earlier Differences in daily food consumption, temperature and salinity may explain large site differences in daily egg production rates.
\end{abstract}

\section{INTRODUCTION}

In sharp contrast to daily vertical migration (e.g. reviews by Cushing 1951 and Bainbridge 1961) and daily feeding (Stearns 1986 and references therein), diel egg laying by copepod zooplankters is a shortinterval, cyclic behavior that is sparsely documented. Although few copepod species have been tested specifically for such behavior, it is becoming clear that dramatic changes in egg production can occur on a time scale of hours (Harding et al. 1951, Marshall \& Orr 1952, Mullin 1968, Spindler 1971, Valentin 1972, Gophen 1978, Saint-Jean \& Pagano 1983, Marcus 1985. Runge 1985). If diel egg laying behavior is common, it would have important consequences for ecological theory dealing with secondary production in aquatic

\footnotetext{
- Present address: The University of Texas at Austin, Marine Science Institute, Port Aransas, Texas 78373-1267, USA

- Addressee for reprint requests
}

systems, because present production coefficients of models do not reflect short-term changes in egg production rate.

The dual purpose of this research was (1) to describe the diel egg-laying pattern of a common calanoid copepod species in 2 estuaries and (2) to determine which environmental factors may be likely candidates as major controlling cues for this cyclic behavior. Acartia tonsa Dana was selected for this research, because of its abundance at the chosen field sites, its ease in handling and evidence of other diel behaviors in this species (Stearns \& Forward 1984, Stearns 1986).

\section{MATERIALS AND METHODS}

Two field experiments were completed in different estuaries to study short-interval changes in egg production by Acartia tonsa. The first experiment (15 to 17 April 1986) took place in the Skidaway River estuary, Savannah, Georgia, USA; the second experiment 
(10 to 12 June 1986) in the Newport River estuary, Beaufort, North Carolina, USA.

During each experiment, copepods were collected periodically from the estuary using a plankton net (202 $\mu \mathrm{m}$ mesh) that was tidally filled from the dock. This method of capture has been found not to affect the timing of egg release by Calanus finmarchicus (Harding et al. 1951) or Calanus pacificus (Runge 1985). Immediately after collection, each sample was taken to a temperature-controlled room $\left( \pm 1 \mathrm{C}^{\circ}\right.$ of field water temperature), where adult female Acartia tonsa were sorted and placed in freshly collected, $20 \mu \mathrm{m}$ or $202 \mu \mathrm{m}$ filtered seawater. Sorting for a given temporal station began $3 \mathrm{~h}$ before deployment in the estuary. Sorting at night was done at low light levels, after which the copepods were left in darkness. Shortly before deployment in the estuary, each adult female was placed in its own $40 \mathrm{ml}$ glass vial filled with freshly collected $20 \mu \mathrm{m}$ or $202 \mu \mathrm{m}$ filtered seawater. Each vial was then tightly capped, turned upside down to avoid an unnatural shadow cast by the lid, placed in a field rack with other copepod vials and lowered into the estuary. Weighted underneath with bricks, the rack was lowered to the bottom of the estuary during daylight hours (depth = 1.5 to $2.8 \mathrm{~m}$ at the April site, 2.5 to $3.4 \mathrm{~m}$ at the June site). At night, the rack was suspended at a depth of $1 \mathrm{~m}$. After ca $3 \mathrm{~h}$ the vials were retrieved and their contents rinsed into separate plastic jars and preserved with $37.4 \%$ formaldehyde solution buffered with borax and mixed with the stain Rose Bengal. Delegating livework procedures to one person, preservation procedures to another and using different rooms for these functions ensured that the experimental copepods were not exposed to preservatives until the appropriate time.

The contents of these jars were later examined for eggs laid by Acartia tonsa. The number of eggs in each vial was divided by the deployment time to estimate the egg production rate (no. of eggs [adult female] ${ }^{-1}$ $\mathrm{h}^{-1}$ ). To correct for predeployment eggs that may have been in the seawater before the copepods were added, correction vials without copepods were filled with the same water, immediately emptied into separate plastic jars and preserved before any hatching could take place. The number of eggs in each of these vials was also divided by the deployment time to provide a correction term in the appropriate units (no. of eggs $\mathrm{h}^{-1}$ ).

Egg abundance in the correction vials containing 202 um filtered seawater (mean no. of eggs per vial for usually 2 replicates during the first experiment, 3 replicates during the second experiment) remained low and did not significantly change from day to night (first field experiment: Model I, 1-way analysis of variance [ANOVA], $F_{\mathrm{s}}=0.124 \mathrm{~ns}, \mathrm{df}=1,11 ;$ grand mean day $=$ 1.14, $\mathrm{SE}=0.210, n=7 ;$ grand mean $_{\text {night }}=1.00, \mathrm{SE}=$
$0.365, n=6$; second field experiment: Model 1, 1-way ANOVA, $F_{\mathrm{s}}=0.212 \mathrm{~ns}$, df $=1,10 ;$ grand mean day $=$ $0.40, \mathrm{SE}=0.170, n=6$; grand mean $_{\text {night }}=0.51, \mathrm{SE}=$ $0.188, n=6$ ). For this reason, egg counts from all $202 \mu \mathrm{m}$ correction samples for each experiment were converted to hourly egg production rates and pooled to provide a grand mean that functioned as the correction factor for that experiment $10.285 \mathrm{eggs}^{-1}$ for the first experiment, $0.122 \mathrm{eggs}^{-1}$ for the second experiment) No correction was made for hatching of predeployment eggs during the deployment period, possibly resulting in a slight underestimation of hourly egg production. Because egg abundance in the correction vials was very low, however, the general conclusions regarding nocturnal egg laying would not have been affected even if hatching of predeployment eggs had occurred only during daylight hours. It is highly unlikely that any of the eggs laid during deployment hatched during that $3 \mathrm{~h}$ period (Ambler 1985, Tester 1985). No correction factor was necessary for the copepod vials containing $20 \mu \mathrm{m}$ filtered seawater, since all eggs were removed by the $20 \mu \mathrm{m}$ mesh screen. Because Acartia tonsa rarely eats its own eggs (Dagg 1977), no correction was made for that factor during these experiments.

For correlative purposes, measurements were made at $3 \mathrm{~h}$ intervals of several field parameters. Light intensity (spectral range $=400$ to $700 \mathrm{~nm}$ ) was measured near the estuarine bottom during daylight hours using either a LI-COR 193S spherical quantum sensor connected to a LI-COR 185 quantum meter (first experiment) or a LI-COR 192S downwelling quantum sensor connected to a LI-COR 185A quantum meter (second experiment). A 2 hp gasoline-driven Teel centrifugal pump (Model 1P855, Dayton Electric Manufacturing Co.) was used to collect unfiltered and filtered (20 $4 \mathrm{~m}$ or 202 um mesh) water near the estuarine bottom during daylight hours and at $1 \mathrm{~m}$ depth at night. Water temperature and salinity were measured immediately. For plant pigment measurements, two $10 \mathrm{ml}$ subsamples were collected from each water sample, filtered, treated with $\mathrm{MgCO}_{3}$ and immediately frozen for later analysis of chlorophyll a and pheopigment a concentrations by fluorescence using a Turner Designs fluorometer (Model 10 series).

Although Acartia tonsa is capable of feeding omnivorously (Turner 1984a), field studies of in situ feeding in coastal environments indicate that the species feeds primarily on phytoplankton (Turner 1984b). For this reason, feeding activity of adult females was estimated by measuring the chlorophyll a and pheopigment a content of nonexperimental copepods periodically collected in the field. During each temporal station, copepod samples were collected in triplicate from near the estuarine bottom during daylight hours and at $1 \mathrm{~m}$ depth at night. Collecting was 
done by serially pumping water from the appropriate depth into 3 plankton nets (158 $\mu \mathrm{m}$ mesh) fitted with clean $125 \mathrm{ml}$ jars. Each sample was processed as described by Stearns (1986), then analyzed with a Turner Designs fluorometer (Model 10 series) for chlorophyll $a$ and pheopigment a concentrations per copepod. At least 10 copepods were used for each sample.

Before any parametric test was done, the data set involved was tested for homoscedasticity using Bartlett's test (Sokal \& Rohlf 1981). Transformations were done when necessary. Parametric tests included computation of the product-moment correlation coefficient, the Model I, 1-way ANOVA and the Student's t-test. The nonparametric Kendall coefficient of rank correlation and the Mann-Whitney $U$-test (Sokal \& Rohlf 1981) were used when parametric testing could not be done. Nonsignificance was determined at $p>0.05$.

\section{RESULTS}

Copepods in the $202 \mu \mathrm{m}$ filtered seawater showed strong nocturnal egg-laying behavior. The corrected mean egg production rate (no. of eggs laid female ${ }^{-1}$ $\mathrm{h}^{-1}$ ) significantly increased at night relative to daytime measurements by a factor of 3.0 during the first field experiment (Fig. 1; Table 1) and 3.9 during the second experiment (Fig. 2; Table 1). Copepods in the $20 \mu \mathrm{m}$ filtered seawater showed a $1.6 \times$ increase in mean egg production rate at night compared with the mean daytime rate. For the June experiment, there was no significant difference in egg production rate between the $202 \mu \mathrm{m}$ and $20 \mu \mathrm{m}$ treatments during daylight hours (Model I, 1-way ANOVA, $F_{\mathrm{s}}=2.905$ ns, $\mathrm{df}=1,9$, see Table 1 for means and standard deviations). A similar comparison showed no significant difference at night, either (Model I, 1-way
Fig. 1. Acartia tonsa field experiment in the Skidaway River estuary, Georgia, USA, 15 to 17 April 1986. Temporal changes in $(A)$ in situ gut fullness of adult females (ng chlorophyll a + pheopigment a copepod ${ }^{-1}, n \geq 10$ copepods per sample, means are connected) and (B) mean egg production rates (no. of eggs female ${ }^{-1} \mathrm{~h}^{-1}$ ) and standard deviations (vertical lines) of copepods retained in separate glass vials containing $202 \mu \mathrm{m}$ filtered seawater, deployed in the estuary for ca 3 h (horizontal lines). Egg production rates were corrected for eggs present in the seawater before each temporal station began. Time in hours (Eastern Standard Time). Dark bars: hours of darkness; $n$ : sample size
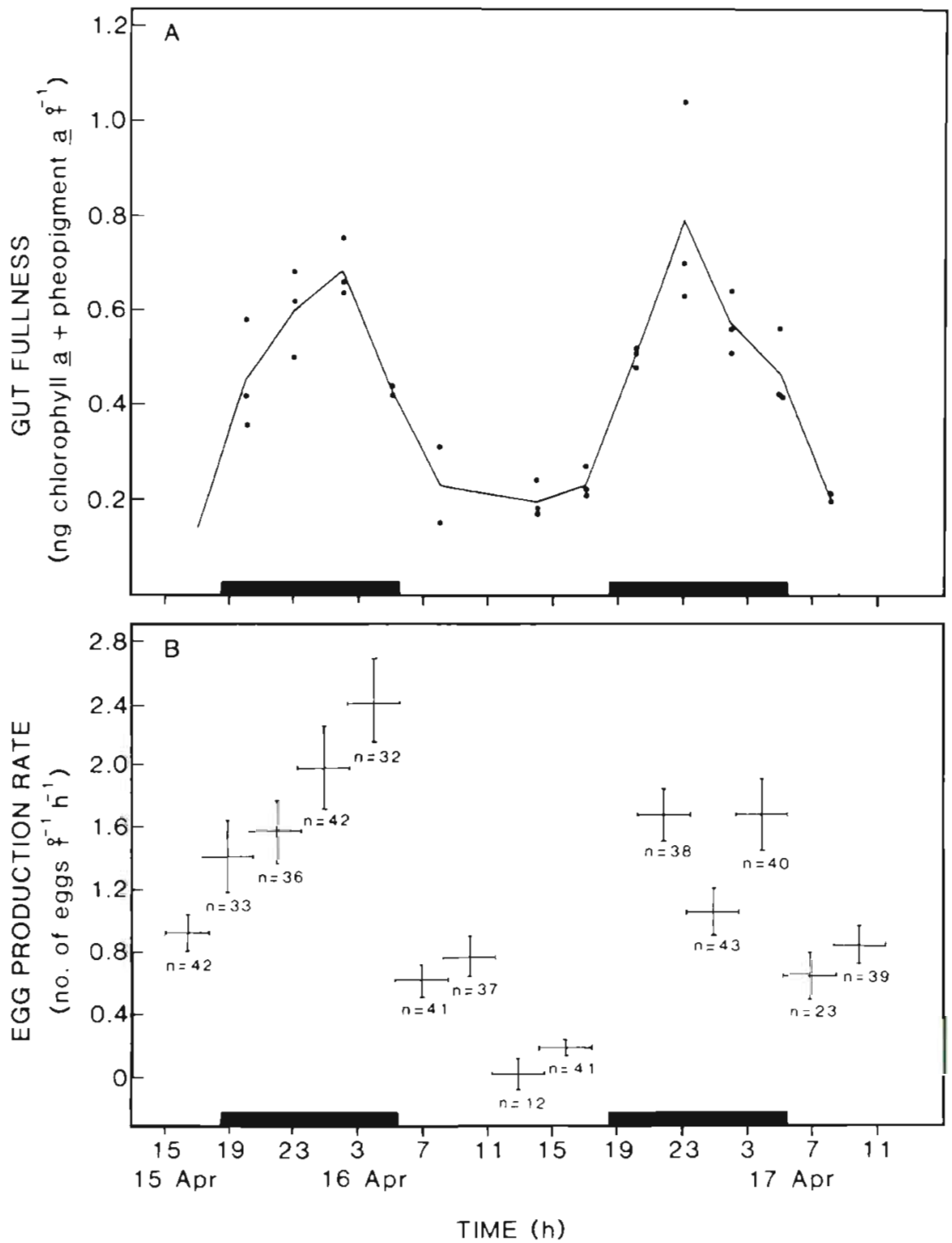

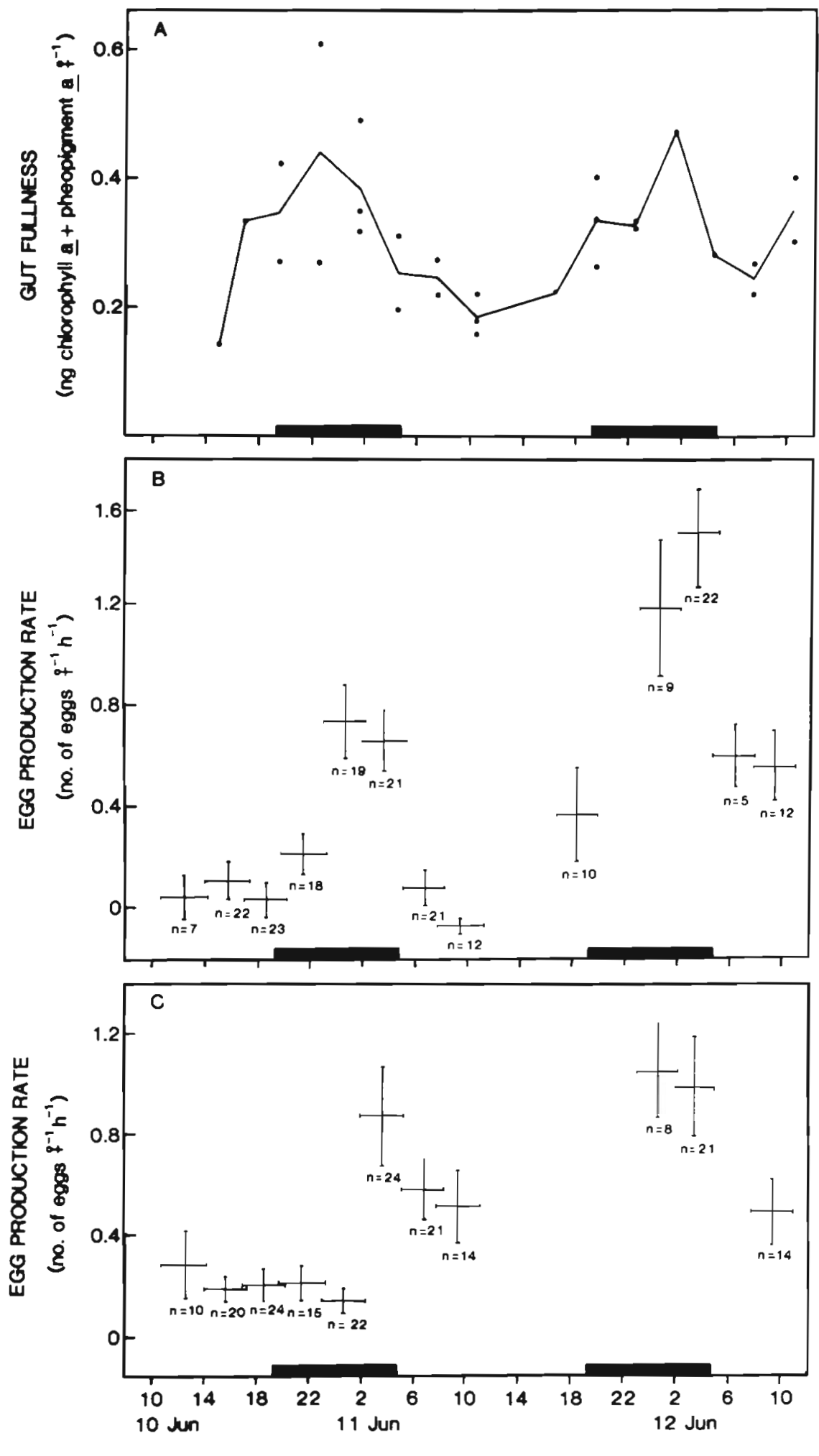

TIME (h)

Fig. 2. Acartia tonsa field experiment in the Newport River estuary, North Carolina, USA, 10 to 12 June 1986. Temporal changes in (A) in situ gut fullness of adult females (ng chlorophyll $a+$ pheopigment a copepod ${ }^{-1}, n \geq 10$ copepods per sample, means are connected) and mean egg production rates (no. of eggs female ${ }^{-1}$ $\mathrm{h}^{-1}$ ) and standard deviations (vertical lines) of copepods retained in separate glass vials containing (B) $202 \mu \mathrm{m}$ filtered and (C) $20 \mu \mathrm{m}$ filtered seawater, deployed in the estuary for ca $3 \mathrm{~h}$ (horizontal lines). Egg production rates for (B) were corrected for eggs present in the seawater before each temporal station began. Time in hours (Eastern Standard Time). Dark bars: hours of darkness; $n$ : sample size 
Table 1. Results of Student's t-test analyses to determine day/night differences in environmental factors during Acartia tonsa field experiments at 2 sites (April: Skidaway River estuary, Georgia, USA; June: Newport River estuary, North Carolina, USA). Egg production data were defined as transitional between day and night and eliminated from these analyses if collected from a $3 \mathrm{~h}$ station that overlapped with sunrise or sunset $\pm 30 \mathrm{~min} .202 \mu \mathrm{m}$ : experiment using $202 \mu \mathrm{m}$ filtered seawater; $20 \mu \mathrm{m}$ : experiment using $20 \mu \mathrm{m}$ filtered seawater; $\mathrm{SD}$ : standard deviation; $n$ : sample size; $t_{\mathrm{s}}$ : $t$-test statistic; $n s$ : not significant $(p>0.05)$

\begin{tabular}{|c|c|c|c|c|c|}
\hline Factor & $\begin{array}{c}\text { Field } \\
\text { experiment }\end{array}$ & $\begin{array}{c}\text { Day } \\
\text { Mean } \pm \mathrm{SD} \quad(n)\end{array}$ & $\begin{array}{l}\text { Night } \\
\text { Mean } \pm \text { SD }\end{array}$ & $\{n\}$ & Result, statistic \\
\hline $\begin{array}{l}\text { Egg productivity } \\
\text { (no. eggs female } \\
\mathrm{h}^{-1} \text { ) }\end{array}$ & $\begin{array}{l}\text { April }(202 \mu \mathrm{m}) \\
\text { June }(202 \mu \mathrm{m}) \\
\text { June }(20 \mu \mathrm{m})\end{array}$ & $\begin{array}{ll}0.58 \pm 0.341 & (7) \\
0.22 \pm 0.284 & (6) \\
0.41 \pm 0.165 & (5)\end{array}$ & $\begin{array}{l}1.72 \pm 0.449 \\
0.85 \pm 0.489 \\
0.65 \pm 0.442\end{array}$ & $\begin{array}{l}(6) \\
(5) \\
(5)\end{array}$ & $\begin{array}{l}\text { Night }>\text { Day; } t_{\mathrm{s}}=5.241(p<0.001) \\
\text { Night }>\text { Day; } t_{\mathrm{s}}=2.695(p=-=0.025) \\
\text { Night }=\text { Day; } t_{\mathrm{s}}=1.145(\mathrm{~ns})\end{array}$ \\
\hline $\begin{array}{l}\text { Gut fullness } \\
\text { (ng chlorophyll a } \\
\text { pheopigment a female }{ }^{-1} \text { ) }\end{array}$ & $\begin{array}{l}\text { April } \\
\text { June }\end{array}$ & $\begin{array}{l}0.20 \pm 0.037 \\
0.25 \pm 0.075\end{array}$ & $\begin{array}{l}0.56 \pm 0.125 \\
0.36 \pm 0.075\end{array}$ & $\begin{array}{l}(8) \\
(8)\end{array}$ & $\begin{array}{l}\text { Night }>\text { Day; } t_{\mathrm{s}}=6.178(p<0.001) \\
\text { Night }>\text { Day; } t_{\mathrm{s}}=2.768(p=0.016)\end{array}$ \\
\hline $\begin{array}{l}\text { Water temperature } \\
\left({ }^{\circ} \mathrm{C}\right)\end{array}$ & $\begin{array}{l}\text { April } \\
\text { June }\end{array}$ & $\begin{array}{rr}20.4 \pm 0.80 & (6) \\
26.4 \pm 0.44 & (11)\end{array}$ & $\begin{array}{l}19.9 \pm 0.56 \\
26.0 \pm 0.46\end{array}$ & $\begin{array}{l}(8) \\
(6)\end{array}$ & $\begin{array}{l}\text { Night }=\text { Day } ; t_{\mathrm{s}}=1.319(\mathrm{~ns}) \\
\text { Night }=\text { Day } ; t_{\mathrm{s}}=1.794(\mathrm{~ns})\end{array}$ \\
\hline Salinity $(\%)$ & $\begin{array}{l}\text { April } \\
\text { June }\end{array}$ & $\begin{array}{l}24.0 \pm 2.00 \\
33.7 \pm 0.51\end{array}$ & $\begin{array}{l}23.1 \pm 1.25 \\
33.7 \pm 0.45\end{array}$ & $\begin{array}{l}(8) \\
(6)\end{array}$ & $\begin{array}{l}\text { Night }=\text { Day; } t_{\mathrm{s}}=1.010(\mathrm{~ns}) \\
\text { Night }=\text { Day; } t_{\mathrm{s}}=0.073(\mathrm{~ns})\end{array}$ \\
\hline $\begin{array}{l}\text { Unfiltered } \\
\text { chlorophyll a }\left(\mu \mathrm{g} \mathrm{l}^{-1}\right)\end{array}$ & $\begin{array}{l}\text { April } \\
\text { June }\end{array}$ & $\begin{array}{lr}5.34 \pm 1.101 & (6) \\
5.96 \pm 1.275 \quad(12)\end{array}$ & $\begin{array}{l}4.40 \pm 0.599 \\
4.20 \pm 1.452\end{array}$ & $\begin{array}{l}(8) \\
(6)\end{array}$ & $\begin{array}{l}\text { Night }=\text { Day; } t_{\mathrm{s}}=2.075(\mathrm{~ns}) \\
\text { Day }>\text { Night } t_{\mathrm{s}}=2.633(p=0.018)\end{array}$ \\
\hline $\begin{array}{l}\text { Unfiltered } \\
\text { pheopigment a } \\
\left(\mu \mathrm{g} \mathrm{l}^{-1}\right)\end{array}$ & $\begin{array}{l}\text { April } \\
\text { June }\end{array}$ & $\begin{array}{rr}4.82 \pm 1.200 \\
5.02 \pm 1.282(12)\end{array}$ & $\begin{array}{l}3.23 \pm 0.320 \\
3.93 \pm 1.161\end{array}$ & $\begin{array}{l}(8) \\
(6)\end{array}$ & $\begin{array}{l}\text { Day }>\text { Night } ; t_{\mathrm{s}}=3.629(p=0.003) \\
\text { Night }=\text { Day; } t_{\mathrm{s}}=1.755 \text { (ns) }\end{array}$ \\
\hline $\begin{array}{l}\text { Unfiltered } \\
\text { chlorophyll a }+ \\
\text { pheopigment } a\left(\mu \mathrm{g} \mathrm{l}^{-1}\right)\end{array}$ & $\begin{array}{l}\text { April } \\
\text { June }\end{array}$ & $\begin{array}{l}10.16 \pm 2.122(6) \\
10.98 \pm 2.409(12)\end{array}$ & $\begin{array}{l}7.62 \pm 0.768 \\
8.13 \pm 2.566\end{array}$ & $\begin{array}{l}(8) \\
(6)\end{array}$ & $\begin{array}{l}\text { Day }>\text { Night } ; t_{s}=3.154(p=0.008) \\
\text { Day }>\text { Night } ; t_{\mathrm{s}}=2.315(p=0.034)\end{array}$ \\
\hline $\begin{array}{l}202 \mu \mathrm{m} \text { filtered } \\
\text { chlorophyll a }\left(\mu \mathrm{g} \mathrm{l}^{-1}\right)\end{array}$ & $\begin{array}{l}\text { April } \\
\text { June }\end{array}$ & $\begin{array}{l}5.53 \pm 1.121 \\
5.88 \pm 1.426(12)\end{array}$ & $\begin{array}{l}4.51 \pm 0.716 \\
4.20 \pm 1.284\end{array}$ & $\begin{array}{l}(8) \\
(6)\end{array}$ & $\begin{array}{l}\text { Night }=\text { Day; } t_{\mathrm{s}}=2.072(\mathrm{~ns}) \\
\text { Day }>\text { Night; } t_{5}=2.432(p=0.027)\end{array}$ \\
\hline $\begin{array}{l}202 \mu \mathrm{m} \text { filtered } \\
\text { pheopigment a } \\
\left(\mu \mathrm{g} \perp^{-1}\right)\end{array}$ & $\begin{array}{l}\text { April } \\
\text { June }\end{array}$ & $\begin{array}{l}4.45 \pm 1.091 \quad(6) \\
5.26 \pm 1.391(12)\end{array}$ & $\begin{array}{l}3.33 \pm 0.578 \\
4.22 \pm 1.497\end{array}$ & $\begin{array}{l}(8) \\
(6)\end{array}$ & $\begin{array}{l}\text { Day }>\text { Night; } t_{\mathrm{s}}=2.506(p=0.028) \\
\text { Night }=\text { Day; } t_{\mathrm{s}}=1.467 \text { (ns) }\end{array}$ \\
\hline $\begin{array}{l}202 \mu \mathrm{m} \text { filtered } \\
\text { chlorophyll } a+ \\
\text { pheopigment } a \\
\left(\mu \mathrm{g} l^{-1}\right)\end{array}$ & $\begin{array}{l}\text { April } \\
\text { June }\end{array}$ & $\begin{aligned} 9.97 & \pm 1.792 \quad(6) \\
11.14 & \pm 2.689(12)\end{aligned}$ & $\begin{array}{l}7.84 \pm 1.149 \\
8.42 \pm 2.770\end{array}$ & $\begin{array}{l}(8) \\
(6)\end{array}$ & $\begin{array}{l}\text { Day }>\text { Night } t_{\mathrm{s}}=2.726(p=0.018) \\
\text { Night }=\text { Day } t_{\mathrm{s}}=2.010(\mathrm{~ns})\end{array}$ \\
\hline $\begin{array}{l}20 \mu \mathrm{m} \text { filtered } \\
\text { chlorophyll } a\left(\mu \mathrm{g} \mathrm{l}^{-1}\right)\end{array}$ & June & $5.44 \pm 1.440(12)$ & $4.17 \pm 1.612$ & (6) & Night $=$ Day $t_{\mathrm{s}}=1.696(\mathrm{~ns})$ \\
\hline $\begin{array}{l}20 \mu \mathrm{m} \text { filtered } \\
\text { pheopigment } a\left(\mu \mathrm{g} \mathrm{l}^{-1}\right)\end{array}$ & June & $4.87 \pm 1.155(12)$ & $3.89 \pm 1.860$ & (6) & Night $=$ Day; $t_{\mathrm{s}}=1.393$ (ns) \\
\hline $\begin{array}{l}20 \mu \mathrm{m} \text { filtered } \\
\text { chlorophyll } a+ \\
\text { pheopigment } a\left(\mu \mathrm{gl}^{-1}\right)\end{array}$ & June & $10.32 \pm 2.505(12)$ & $8.06 \pm 3.334$ & (6) & Night $=$ Day $_{i} t_{\mathrm{s}}=1.615(\mathrm{~ns})$ \\
\hline
\end{tabular}

ANOVA, $F_{\mathrm{s}}=0.450 \mathrm{~ns}$, df $=1,8$, see Table 1 for means and standard deviations).

For each field experiment, the possible relation between temporal changes in egg production rate and changes in each of the measured environmental factors was examined. Day/night differences in egg production obviously correlated with day/night differences in light intensity. Highest light levels recorded near the estuarine bottom were 296.8 and $62.5 \mu \mathrm{E} \mathrm{s}^{-1} \mathrm{~m}^{-2}$ during the April and June field experiments, respectively. Water temperature and salinity did not show signifi- cant day/night differences (Table 1) and no significant correlations were found between each of these 2 factors [(value at the beginning of the temporal station + value at its end)/2] and egg production rate (Table 2). Plant pigment concentrations (chlorophyll $a$, pheopigment $a$, combined pigments) showed either no significant day/ night difference or were significantly higher during daylight hours when both ingestion and egg production rates were lowest (Table 1). Correlation coefficients relating each plant pigment group [(mean concentration at the beginning of each temporal station + 
Table 2. Correlation coefficients showing the association between egg production rate (no. of eggs female $\mathrm{h}^{-1} \mathrm{~h}^{-1}$ ) and each of several factors during Acartia tonsa field experiments at the 2 sites. To reduce variance resulting from less accurate estimates of association during twilight periods, transitional stations (defined in Table 1) were eliminated. Some symbols are defined in Table 1. $r$ : product-moment correlation coefficient; $\tau$ : Kendall coefficient of rank correlation; ${ }^{a} \log$ transformation; ${ }^{b} \log (x+1)$ transformation

\begin{tabular}{|c|c|c|c|c|}
\hline Factor & Field experiment & Result & Significance & $n$ \\
\hline $\begin{array}{l}\text { Gut fullness } \\
\text { (ng chlorophyll } a+ \\
\text { pheopigment a } \\
\text { female }^{-1} \text { ) }\end{array}$ & $\begin{array}{l}\text { April }(202 \mu \mathrm{m}) \\
\text { June }(202 \mu \mathrm{m}) \\
\text { June }(20 \mu \mathrm{m})\end{array}$ & $\begin{aligned} a_{r} & =0.899 \\
\tau & =0.600 \\
\tau & =0.111\end{aligned}$ & $\begin{array}{l}p<0.001 \\
p<0.05 \\
\text { ns }\end{array}$ & $\begin{array}{r}9 \\
10 \\
9\end{array}$ \\
\hline $\begin{array}{l}\text { Water temperature } \\
\left({ }^{\circ} \mathrm{C}\right)\end{array}$ & $\begin{array}{l}\text { April }(202 \mu \mathrm{m}) \\
\text { June }(202 \mu \mathrm{m}) \\
\text { June }(20 \mu \mathrm{m})\end{array}$ & $\begin{array}{l}r=-0.118 \\
r=-0.376 \\
r=-0.435\end{array}$ & $\begin{array}{l}\text { ns } \\
\text { ns } \\
\text { ns }\end{array}$ & $\begin{array}{l}11 \\
11 \\
10\end{array}$ \\
\hline Salinity $(\%)$ & $\begin{array}{l}\text { April }(202 \mu \mathrm{m}) \\
\text { June }(202 \mu \mathrm{m}) \\
\text { June }(20 \mu \mathrm{m})\end{array}$ & $\begin{array}{l}r=-0.279 \\
r=-0.086 \\
r=-0.474\end{array}$ & $\begin{array}{l}\text { ns } \\
\text { ns } \\
\text { ns }\end{array}$ & $\begin{array}{l}11 \\
11 \\
10\end{array}$ \\
\hline $\begin{array}{l}\text { Unfiltered } \\
\text { chlorophyll a } \\
\left(\mu \mathrm{g} \mathrm{l}^{-1}\right)\end{array}$ & $\begin{array}{l}\text { April }(202 \mu \mathrm{m}) \\
\text { June }(202 \mu \mathrm{m}) \\
\text { June }(20 \mu \mathrm{m})\end{array}$ & $\begin{aligned} r & =-0.473 \\
\mathrm{~b}_{r} & =-0.677 \\
\tau & =0.022\end{aligned}$ & $\begin{array}{l}\text { ns } \\
p<0.05 \\
\text { ns }\end{array}$ & $\begin{array}{l}11 \\
11 \\
10\end{array}$ \\
\hline $\begin{array}{l}\text { Unfiltered } \\
\text { pheopigment a } \\
\left(\mu \mathrm{I} \mathrm{I}^{-1}\right)\end{array}$ & $\begin{array}{l}\text { April }(202 \mu \mathrm{m}) \\
\text { June }(202 \mu \mathrm{m}) \\
\text { June }(20 \mu \mathrm{m})\end{array}$ & $\begin{array}{l}r=-0.782 \\
r=-0.653 \\
\tau=-0.111\end{array}$ & $\begin{array}{l}p<0.005 \\
p<0.05 \\
\text { ns }\end{array}$ & $\begin{array}{l}11 \\
11 \\
10\end{array}$ \\
\hline $\begin{array}{l}\text { Unfiltered } \\
\text { chlorophyll a }+ \\
\text { pheopigment a } \\
\left(\text { ug l}^{-1} \text { ) }\right.\end{array}$ & $\begin{array}{l}\text { April }(202 \mu \mathrm{m}) \\
\text { June }(202 \mu \mathrm{m}) \\
\text { June }(20 \mu \mathrm{m})\end{array}$ & $\begin{aligned} r & =-0.739 \\
{ }^{b} r & =-0.693 \\
\tau & =-0.022\end{aligned}$ & $\begin{array}{l}p<0.01 \\
p<0.02 \\
\text { ns }\end{array}$ & $\begin{array}{l}11 \\
11 \\
10\end{array}$ \\
\hline $\begin{array}{l}202 \text { um filtered } \\
\text { chlorophyll } a \\
\left(\mu \mathrm{gl}^{-1}\right)\end{array}$ & $\begin{array}{l}\text { April }(202 \mu \mathrm{m}) \\
\text { June }(202 \mu \mathrm{m})\end{array}$ & $\begin{array}{l}r=-0.521 \\
r=-0.802\end{array}$ & $\begin{array}{l}\mathrm{ns} \\
p<0.005\end{array}$ & $\begin{array}{l}11 \\
11\end{array}$ \\
\hline $\begin{array}{l}202 \mu \mathrm{m} \text { filtered } \\
\text { pheopigment a } \\
\left(\mu \mathrm{g} \mathrm{l}^{-1}\right)\end{array}$ & $\begin{array}{l}\text { April }(202 \mu \mathrm{m}) \\
\text { June }(202 \mu \mathrm{m})\end{array}$ & $\begin{array}{l}r=-0.849 \\
r=-0.815\end{array}$ & $\begin{array}{l}p<0.001 \\
p<0.005\end{array}$ & $\begin{array}{l}11 \\
11\end{array}$ \\
\hline $\begin{array}{l}202 \mu \mathrm{m} \text { filtered } \\
\text { chlorophyll } a+ \\
\text { pheopigment } a \\
\left(\mu \mathrm{g} \mathrm{l}^{-1}\right)\end{array}$ & $\begin{array}{l}\text { April }(202 \mu \mathrm{m}) \\
\text { June }(202 \mu \mathrm{m})\end{array}$ & $\begin{array}{l}r=-0.742 \\
b_{r}=-0.848\end{array}$ & $\begin{array}{l}p<0.01 \\
p<0.001\end{array}$ & $\begin{array}{l}11 \\
11\end{array}$ \\
\hline $\begin{array}{l}20 \mu \mathrm{m} \text { filtered } \\
\text { chlorophyll a } \\
\left(\mu \mathrm{g} l^{-1}\right)\end{array}$ & June $(20 \mu \mathrm{m})$ & $\tau=-0.200$ & ns & 10 \\
\hline $\begin{array}{l}20 \mu \mathrm{m} \text { filtered } \\
\text { pheopigment } a \\
\left(\mu \mathrm{g} \mathrm{l^{-1 } )}\right.\end{array}$ & June $(20 \mu \mathrm{m})$ & $\tau=-0.244$ & ns & 10 \\
\hline $\begin{array}{l}20 \mu \mathrm{m} \text { filtered } \\
\text { chlorophyll } a+ \\
\text { pheopigment } a \\
\left(\mu \mathrm{g} \mathrm{l}^{-1}\right)\end{array}$ & June $(20 \mu \mathrm{m})$ & $\tau=-0.200$ & ns & 10 \\
\hline
\end{tabular}

mean concentration at its end)/2] with egg production rate were therefore either not significant or significant but negative (Table 2).

In contrast to these environmental factors, copepod gut fullness increased significantly at night by a factor of 2.8 over the mean daytime value during the first field experiment (Fig. 1, Table 1) and 1.4 during the second experiment (Fig. 2, Table 1). A significant, positive correlation was found between copepod gut fullness in the field l(mean gut fullness at the beginning of the temporal station + mean gut fullness at its end)/2] and the rate of egg production by copepods in $202 \mu \mathrm{m}$ filtered seawater, whereas no such significant correlation was found for copepods in $20 \mu \mathrm{m}$ filtered seawater (Table 2).

For each field experiment, the correlation was calculated between in situ copepod gut fullness and egg production measured at the same time (time lag $=0$ ). 
Similar correlations were calculated at different time intervals between copepod gut fullness and subsequent measurements of egg production. The resulting correlations are shown in Fig. 3 . The strong negative correlations found at time lag 12 h result from the diel feeding and diel egg-production cycles' being strongly out of phase when shifted from each other by that time interval. For copepods in $202 \mu \mathrm{m}$ filtered seawater, egg production positively correlated highest with gut fullness measured at the same time and 24 to 27 h earlier. For copepods in $20 \mu \mathrm{m}$ filtered seawater, the only strongly positive correlations occurred when the time lag was 24 to 27 h. These results suggest that copepod egg production is influenced by the degree of feeding that occurred 24 to 27 h earlier.

The 2 estuarine experiments are compared in Table 3. The April experiment showed markedly higher daily egg production, significantly higher copepod gut fullness, significantly lower water temperature and significantly lower salinity than the June experiment. The plant pigment concentrations were similar although somewhat lower in April than in June.

\section{DISCUSSION}

There have been only a few experiments that have tested for nocturnal egg laying by zooplankton. As the conditions for these experiments have approached those found in nature, evidence of this diel behavior has become stronger [e.g. compare results of Valentin (1972), Uye (1981) and Saint-Jean \& Pagano (1983) for Acartia clausi; Parrish \& Wilson (1978) and this research for Acartia tonsa]. Nocturnal egg laying may be as common a component of planktonic communities as other cyclic behaviors like nocturnal vertical migration and nocturnal feeding. A. tonsa is often a dominant species in estuarine and coastal systems of North and South America, Australia and the countries bordering on the Indian Ocean (Raymont 1983). By documenting nocturnal egg-laying behavior in such a common copepod, this research generalizes the occurrence and therefore relevance of this cyclic behavior in coastal planktonic communities. More research is needed, however, to assess properly the general nature of nocturnal egg laying.

Little research has been directed towards understanding how diel egg-laying behavior is timed. To our knowledge, no one has considered natural, short-term changes in water temperature, salinity and plant pigment concentrations and their possible association with short-interval changes in zooplankton egg production rates during a $24 \mathrm{~h}$ period, although Durbin et al. (1983) did find a significant, positive correlation between the daily egg production rate of Acartia tonsa and food

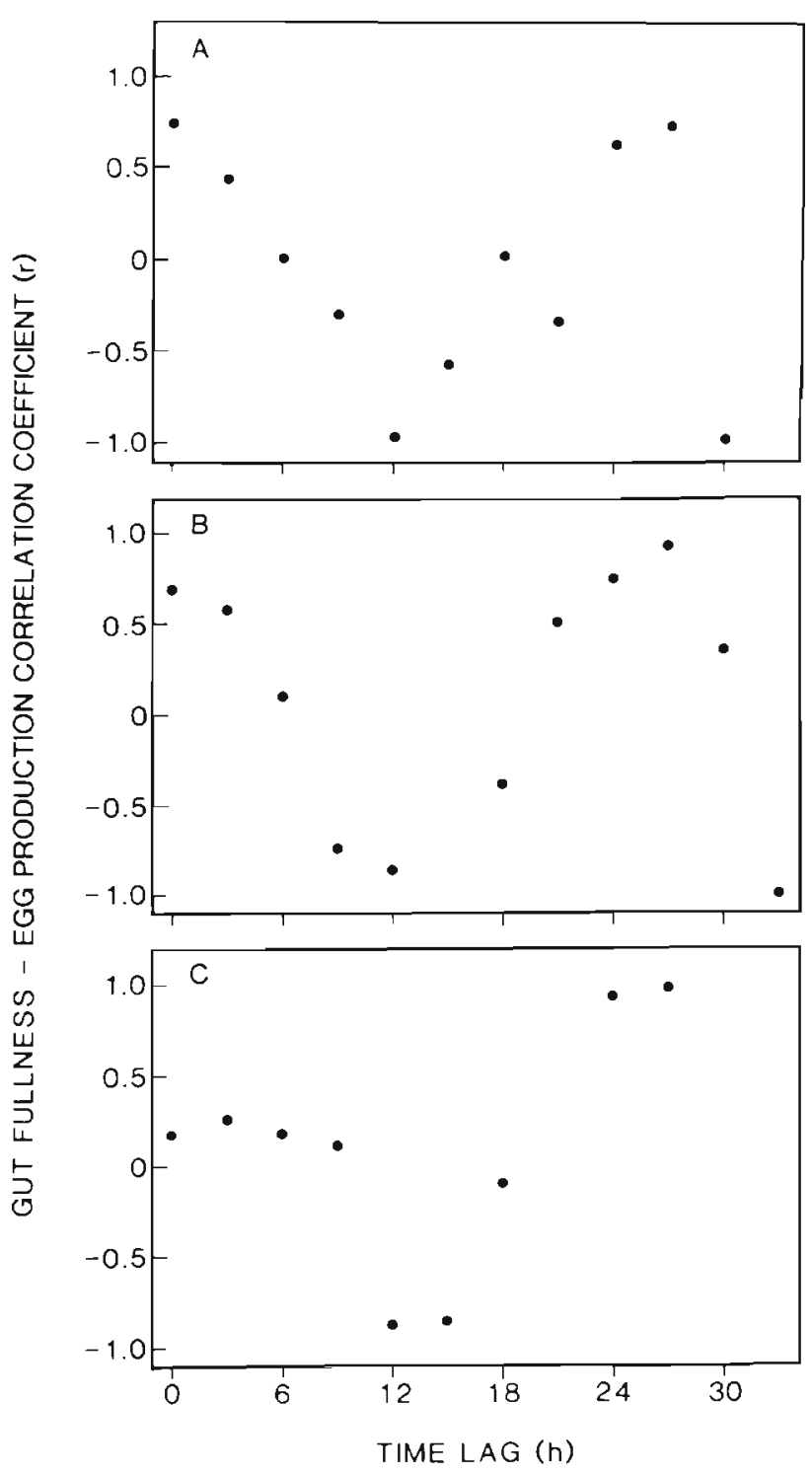

Fig. 3. Acartia tonsa. Product-moment correlation coefficients associating mean in situ gut fullness of adult females (ng chlorophyll $a+$ pheopigment a copepod ${ }^{-1}$ ) and egg production rate (no. of eggs female $\mathrm{h}^{-1} \mathrm{~h}^{-1}$ ) measured at different intervals between feeding and subsequent egg production for copepods deployed in the estuary in separate glass vials containing $202 \mu \mathrm{m}$ filtered seawater (A: Skidaway River; B: Newport River) or $20 \mu \mathrm{m}$ filtered seawater (C: Newport River)

availability (chlorophyll a) during the preceding 24 to $48 \mathrm{~h}$. We found no evidence suggesting that any of these 3 factors was a timing cue directly shaping the nocturnal pattern of egg laying in A. tonsa during the 2 field experiments.

There is evidence that the copepods in $20 \mu \mathrm{m}$ filtered seawater during sorting and deployment in the estuary were deprived of the food size range within which they show the highest clearance rate and greatest efficiency of food capture (Bartram 1981). Nevertheless, these 
Table 3. Comparison between field sites (April: Skidaway River estuary; June: Newport River estuary) for different factors relating to Acartia tonsa field experiments. Daily egg production was calculated by multiplying each mean hourly rate by the appropriate hourly interval, then summing over each of two $24 \mathrm{~h}$ periods. Daily food consumption was similarly calculated, following the assumption that gut contents were removed and replaced every $30 \mathrm{~min}$ (Stearns et al. 1987). Missing data during the second day of each experiment were replaced with data collected $24 \mathrm{~h}$ earlier Some symbols are defined in Table $1 U_{\mathrm{s}}$ : statistic for MannWhitney $U$-test

\begin{tabular}{|c|c|c|c|c|c|}
\hline Factor & $\begin{array}{l}\text { April }(202 \mu \mathrm{m}) \\
\text { Mean } \pm \mathrm{SD}\end{array}$ & $(n)$ & $\begin{array}{l}\text { June }(202 \mu \mathrm{m}) \\
\text { Mean } \pm \mathrm{SD}\end{array}$ & $(n)$ & Result; statistic \\
\hline $\begin{array}{l}\text { Daily egg production } \\
\text { (no. eggs female } \\
d^{-1} \text { ) }\end{array}$ & $25.5 \pm 3.54$ & (2) & $10.0 \pm 5.66$ & $(2)$ & April $=$ June $_{i} t_{\mathrm{s}}=3.286$ (ns) \\
\hline $\begin{array}{l}\text { Unfiltered } \\
\text { chlorophyll a }\left(\mu \mathrm{g} \mathrm{l}^{-1}\right)\end{array}$ & $4.8 \pm 0.95$ & (14) & $5.4 \pm 1.55$ & $(18)$ & April $=$ June $_{i} t_{5}=1.274(\mathrm{~ns})$ \\
\hline $\begin{array}{l}\text { Unfiltered } \\
\text { pheopigment a }(\mu \mathrm{g} \\
\left.\mathrm{l}^{-1}\right)\end{array}$ & $3.9 \pm 1.13$ & (14) & $4.7 \pm 1.32$ & $(18)$ & April $=$ June $_{i} t_{\mathrm{s}}=1.809$ (ns) \\
\hline $\begin{array}{l}\text { Unfiltered } \\
\text { chlorophyll } a+ \\
\text { pheopigment a ( } \mu \mathrm{g} \\
\left.\mathrm{I}^{-1}\right)\end{array}$ & $8.7 \pm 1.94$ & (14) & $10.0 \pm 2.76$ & $(18)$ & April $=$ June $; t_{s}=1.496(\mathrm{~ns})$ \\
\hline $\begin{array}{l}\text { Daily tood consump- } \\
\text { tion } \\
\text { (ng chlorophyll a } \\
\text { pheopigment a } \\
\text { [adult female }]^{-1} \mathrm{~d}^{-1} \text { ) }\end{array}$ & $18.5 \pm 0.71$ & (2) & $14.1 \pm 0.14$ & $(2)$ & April $>$ June; $t_{s}=8.63(p=0.013)$ \\
\hline $\begin{array}{l}\text { Water temperature } \\
\left({ }^{\circ} \mathrm{C}\right)\end{array}$ & $20.1 \pm 0.69$ & $(14)$ & $26.2 \pm 0.47$ & $(18)$ & April $<$ June $_{i} t_{\mathrm{s}}=29.77(p<0.001)$ \\
\hline Salinity $(\% 1 \%)$ & $23.5 \pm 1.61$ & (14) & $33.7 \pm 0.47$ & (18) & April $<$ June; $U_{s}=126(p<0.001)$ \\
\hline
\end{tabular}

specimens showed the same general results regarding nocturnal egg production as those kept in the better food environment of $202 \mu \mathrm{m}$ filtered seawater. Any possible immediate effect of deployment feeding conditions on egg production would have been cumulative throughout the experiment, yet there was no real difference in daily egg production (no. of eggs female f $^{-1}$ $\mathrm{d}^{-1}$ ) between the 2 June treatments (Model I, 1-way ANOVA, $F_{\mathrm{s}}=0.014 \mathrm{~ns}, \mathrm{df}=1,2 ;$ mean $_{202 \mathrm{\mu m}}=10.0$, $\mathrm{SE}=4.00, n=2 ; \operatorname{mean}_{20 \mu \mathrm{m}}=10.5, \mathrm{SE}=1.50, n=2$ ). These results indicate that the feeding state of Acartia tonsa at a particular moment does not immediately affect its egg production rate.

While there are experimental results that suggest an endogenous rhythm in egg-laying behavior (Spindler 1971. Saint-Jean \& Pagano 1983, Marcus 1985), the same findings could also be explained by an endogenous rhythm in feeding (e.g. Duval \& Geen 1976 , Stearns 1986), with cyclic egg production the resulting by-product of cyclic changes in ingested food available for such production. Gophen (1978) explained higher egg production by Mesocyclops leuckarti in constant darkness than in alternated light and dark periods as the result of higher feeding activity in darkness, with a time lag of ca $1 \mathrm{~d}$ between feeding and subsequent egg production within the experimental temperature range
(22 to $27^{\circ} \mathrm{C}$ ). We found Acartia tonsa to be both a nocturnal grazer and a nocturnal egg layer (Figs. 1 and 2) with highly positive correlations between these 2 behaviors when the time lag is 24 to $27 \mathrm{~h}$ (Fig. 3). Research is presently being done to determine whether a diel feeding rhythm in this species (Stearns 1986) directly controls the diel timing of egg-laying behavior and whether there is an independent, endogenous rhythm in the egg production rate.

Mullin (1968) tested for the direct effects of light on spawning in female Calanus helgolandicus and concluded that the general nocturnal egg-laying pattern was partly but not entirely shaped by light near sunrise. Similarly, Valentin (1972) found a large difference between the diel egg-laying pattern of Centropages typicus exposed to light under laboratory conditions and that of individuals treated similarly but kept in darkness. We are presently investigating the possible roles of light as a direct effector or as a Zeitgeber for an endogenous, egg-laying rhythm in Acartia tonsa.

Although nocturnal egg-laying behavior in Acartia tonsa was found in both estuaries, markedly higher daily egg production occurred during the April experiment in the Skidaway River estuary than during the June experiment in the Newport River estuary (Table 3). Several factors may help explain this result. Because 
A. tonsa does not rely heavily on stored food reserves (Dagg 1977), its egg production is closely associated with feeding within the past day (Fig. 3). Daily food consumption was significantly higher during the April experiment than during the June experiment (Table 3), perhaps explaining the site differences in daily egg production.

Nights were longer during the April experiment than during the June experiment, so maximal egg production rates continued over a larger fraction of the $24 \mathrm{~h}$ day in April than in June. However, regardless of day/ night phase, hourly production rates were more than twice as high in April than in June (Table 1), so factors not associated with photoperiod must also be involved.

Water temperature was significantly higher in June $\left(26.2^{\circ} \mathrm{C}\right.$ ) than in April $\left(20.1^{\circ} \mathrm{C}\right)$. For a poikilotherm like Acartia tonsa, higher water temperature may result in a higher metabolic rate with more energy being used for metabolic maintenance and consequently less energy available for egg production. For adult $A$. tonsa fed algal cultures at saturating concentrations, Ambler (1985) found higher daily production at ca $20^{\circ} \mathrm{C}$ than at higher temperatures, supporting this explanation. A lower daily ration would further decrease egg production in June.

Salinities during the June experiment were significantly higher than those during the April experiment (Table 3). There is evidence that $30 \%$ is suboptimal for maximal growth of Acartia tonsa compared with lower salinities (Heinle 1981). Ambler (1985) reported increasing daily egg production of this species with decreasing salinities ( 30 to $10 \%$ ). These salinity effects may help explain the difference in daily egg production found during these 2 field experiments.

This research describes a sparsely documented but possibly common zooplankton phenomenon: nocturnal egg-laying behavior. There is no evidence that water temperature, salinity or in situ plant pigment concentration controls the diel timing of this behavior in Acartia tonsa. However, there is a relation between feeding and hourly egg production rates 24 to 27 h later, suggesting that cyclic feeding behavior may be important in controlling the timing of nocturnal egg laying. More research is needed to distinguish among possible timing cues like endogenous behavior, nocturnal feeding and the direct effects of light. While nocturnal egglaying behavior was found in both field experiments, the two differed in daily egg production in ways that can be explained by differences in daily food consumption, photoperiod, water temperature and salinity.

Acknowledgements. Funding for this research was provided by the Skidaway Institute of Oceanography and the Dauphin Island Sea Lab. We thank the following people for use of equipment: G.-A. Paffenhöfer (Skidaway Institute of Oceanography), J. Richardson (Savannah State College), J.
Sutherland (Duke University Marine Laboratory), G. Thorpe (Duke University Marine Laboratory) and J. Yoder (Skidaway Institute of Oceanography). We also thank M. Murdoch for translation of key articles, D. Colby for statistical advice, $\mathrm{H}$. Garrett for typing of the manuscript, H. Gordy for preparation of the figures and $\mathrm{H}$. Nearing for preparation of the tables. We further thank 3 anonymous reviewers for constructive criticism of the manuscript. Offprint expenses were covered by the National Marine Fisheries Service, Beaufort, North Carolina, USA. This article is dedicated to Edison Alfred Stearns.

\section{LITERATURE CITED}

Ambler, J. W. (1985). Seasonal factors affecting egg production and viability of eggs of Acartia tonsa Dana from East Lagoon, Galveston, Texas. Estuar. coast. Shelf Sci. 20: $743-760$

Bainbridge, R. (1961). Migrations. In: Waterman, T. H. (ed.) The physiology of Crustacea, Vol. II, Sense organs, integration, and behavior Academic Press, New York, p. $431-463$

Bartram, W. C. (1981). Experimental development of a model for the feeding of neritic copepods on phytoplankton. J. Plankton Res. 3: 25-51

Cushing, D. H. (1951). The vertical migration of planktonic Crustacea. Biol. Rev. 26: 158-192

Dagg, M. (1977). Some effects of patchy food environments on copepods. Limnol. Oceanogr. 22: 99-107

Durbin, E. G., Durbin, A. G., Smayda, T. J., Verity, P. S. (1983). Food limitation of production by adult Acartia tonsa in Narragansett Bay, Rhode Island. Limnol. Oceanogr. 28: $1199-1213$

Duval, W. S., Geen, G. H. (1976). Diel feeding and respiration rhythms in zooplankton. Limnol. Oceanogr 21: 823-829

Gophen, M. (1978). Errors in the estimation of recruitment of early stages of Mesocyclops leuckarti (Claus) caused by the diurnal periodicity of egg-production. Hydrobiologia 57: $59-64$

Harding, J. P., Marshall, S. M., Orr, A. P. (1951). Time of egglaying in the planktonic copepod Calanus. Nature, Lond. 167: 953

Heinle, D. R. (1981). Zooplankton. In: Vernberg, F. J., Vernberg, W. B. (eds.) Functional adaptations of marine organisms. Academic Press, New York, p. 85-145

Marcus, N. H. (1985). Endogenous control of spawning in a marine copepod. J. exp. mar. Biol. Ecol. 91. 263-269

Marshall, S. M., Orr, A. P. (1952). On the biology of Calanus finmarchicus. VII. Factors affecting egg production. J. mar. biol. Ass. U.K. 30: 527-547

Mullin, C. H. (1968). Egg-laying in the planktonic copepod Calanus helgolandicus (Claus). Crustaceana Suppl. 1: 29-34

Parrish, K. K., Wilson, D. F. (1978). Fecundity studies on Acartia tonsa (Copepoda: Calanoida) in standardized culture. Mar. Biol. 46: 65-81

Raymont, J. E. G. (1983). Plankton and productivity in the oceans, Vol. 2, Zooplankton, 2nd edn. Pergamon Press, New York

Runge, J. A. (1985). Relationship of egg production of Calanus pacificus to seasonal changes in phytoplankton availability in Puget Sound, Washington. Limnol. Oceanogr. 30: $382-396$

Saint-Jean, L., Pagano, M. (1983). Rythme journalier de ponte chez Acartia clausi en lagune Ebrié (Côte d'Ivoire). Rev. Hydrobiol. Trop. 16: 145-150 
Sokal, R. R., Rohlf, F. J. (1981). Biometry, 2nd edn. W. H. Freeman, New York

Spindler, K.-D. (1971). Der Einfluß von Licht auf die Eiablage des Copepoden Cyclops vicinus. Z. Naturforsch. Teil B 26 : 953-955

Stearns, D. E. (1986). Copepod grazing behavior in simulated natural light and its relation to nocturnal feeding. Mar Ecol. Prog. Ser. 30: 65-76

Stearns, D. E., Forward, R. B., Jr (1984). Copepod photobehavior in a simulated natural light environment and its relation to nocturnal vertical migration. Mar. Biol. 82: 91-100

Stearns, D. E., Litaker, W., Rosenberg, G. (1987). Impacts of zooplankton grazing and excretion on short-interval fluctuations in chlorophyll $a$ and nitrogen concentrations in a well-mixed estuary. Estuar. coast. Shelf Sci. 24: 305-325

Tester, P. A. (1985). Effects of parental acclimation temperature

This article was submitted to the editor and egc-incubation temperature on egg-hatching time in Acartia tonsa (Copepoda: Calanoida). Mar. Biol. 89: 45-53

Tumer, J. T. (1984a). The feeding ecology of some zooplankters that are important prey items of larval fish. NOAA Tech. Rep. NMFS 7: 1-28

Tumer, J. T (1984b). Zooplankton feeding ecology: contents of fecal pellets of the copepods Acartia tonsa and Labidocera aestiva from continental shelf waters near the mouth of the Mississippi River P.S.Z.N.I.: Mar. Ecol. 5: 265-282

Uye, S.-I. (1981). Fecundity studies of neritic calanoid copepods Acartia clausi Giesbrecht and A. steueri Smirnov: a simple empirical model of daily egg production. J. exp. mar. Biol. Ecol. 50: 255-271

Valentin, J. (1972). La ponte et les oeufs chez les copépodes du Golfe de Marseille: cycle annuel et étude expérimentale. Téthys $4: 349-390$

Manuscript received: May 10, 1988

Revised version accepted: November 17, 1988 